\title{
Human Gender Classification using Machine Learning
}

\author{
Miss. Vaishnavi Y. Mali \\ Student of Electronics Department \\ Walchand College of Engineering \\ Sangli, Indi.
}

\author{
Dr. Babasaheb G. Patil \\ Professor of Electronic Department \\ Walchand College of Engineering \\ Sangli, India.
}

\begin{abstract}
Human Gender classification is one of the most interested and critical area of research. Research contains interactions between computers and human which includes vast information concerning difference in characteristics of males and females. In several kind of pattern recognition, machine learning gives a relation between gender and face. This paper proposes comparison between different techniques used for gender classification. Face is a unique biometric feature of the individual. Facial images with different combinations including frontal, aligned, smiling, non-smiling as well as expression images make the system complicated. Various face recognition methods such as Convolutional neural networks, Delaunay triangulations, geometry based methods like SVM (Support vector machine), LDA (Linear discriminant analysis).For human gender classification, SVM provides better accuracy as compared with existing methods.
\end{abstract}

Keywords- Gender Classification; Face recognition; Machine Learning

\section{INTRODUCTION}

In era of Artificial intelligence, image processing and machine learning plays vital role. Over the period of time, gender classification has obtained wide importance. The aim of paper is to classify the human according to characteristic differences. The different areas of applications are identified where gender classification is important.

Now a day, artificial intelligence for automated machines like robots are most important since it is essential for human. Human can classify gender easily but it is challenging task for automated machines. Our brain stores the details of object in terms of lines, edges or some patterns and remembers the same to find the information for classification.

A security entrance can use gender information for surveillance and control to some area. . For example, female meeting is scheduled in conference hall and task is to allow only females to access the conference hall. It is spare application area which aid in restricted to one sex only. Another application of gender classification is in commercial area. An electronic billboard machine is the best example to display ads. Depending upon the gender detected at billboard, billboard machine choose the advertisement and display on flat panel cards. For effective service to individual, it is required to have more information about their sex. Hence task is to retrieve more accuracy when image quality is poor.

\section{LITURATURE REVIEW}

Many research papers that are related to gender classification for different types of databases have been surveyed to identify numerous approaches use for different gender classification problems. In addition, the different classification analyses that are described in research paper have been viewed to get ideas on how the classification is treated.

Sandeep Kumar in [1] has proposed the gender recognition algorithm using face images. For preprocessing, Brightness of an image Preserving Dynamic Fuzzy Histogram Equalization (BPDFHE) utilized. In this paper, Scale Invariant Fourier Transform (SIFT) was used for feature extraction and Support Vector Machine (SVM) was applied for classification. Performance results were compared on live images, FEI and SCIEN databases and obtained accuracy $98 \%, 91 \%$ and $94 \%$ respectively.

The paper [2] represents facial attribute recognition by recurrent learning with visual fixation. Face features were computed with $\mathrm{CNN}$ and development of features was encoded with a recurrent network. The visual fixation was generally region between eyes or on the eyes and expanded to nose and lips. Fixation was extended to facial attribute using videos also. Proposed system obtained recognition rate of 89.8 using ADIENCE dataset and 91.36 using MULTI-PIE dataset.

Recognition of face by computers gives more accuracy as compared with human. Because of redundant and feature equality, feature selection is complex problem. The proposed method by J.E.Tapia and C.A.Perez[3] removes unrelated feature and applied to refine classification accuracy. Fusing a filter and a wrapper quantified correlation among features and selected clusters formed using mutual information for classification. SVM classifier is used to classify the gender of the person. Proposed method tested using nine datasets gave more accuracy as compared to CNN classifier.

The paper [4] proposed a best method to recognize the gender by using face images. Dataset contains people in regular walking motion and two algorithms deal with face images extracted from videos. Viola-Jones algorithm applied on the input images to detect face and after that face alignment algorithm used for pre-processing. During face recognition, fusion of trainable and domain specific features were used. The COSFIRE and the SURF-based descriptors 
represent different properties of human face which distinguished gender of the human being. The COSFIREbased method achieved more accuracy in comparison to SURF-based method.

For physician, it is an appreciable challenge to identify gender of individual brain. In this paper [5], three dimensional feature descriptor and weighted histogram of gradient orientation (3DWHGO) were proposed to express complex structure of brain. Classification of gender was based on MRI 3D images and described to test feasibility of classifying gender. Proposed method gave more accuracy as compared to 3D SIFT and PCA approach. But this method is hopeless in case of accident which damages the brain.

Due to low cost of SMS (Short Message Service) has become supportive way for communication. Identifying gender and age from SMS text messages is challenging task due to vast vocabulary. In paper [6], 38,588 text messages were tested which includes text written in English and Singaporean English language. For identification of gender, SVM, Naïve Bayes and J48 algorithms was applied and obtained accuracies $78.34 \%, 66.59 \%$ and $79.10 \%$ respectively.

Different approaches for detecting the gender of the person based on various features. The paper[7] introduced same iris code for gender prediction and recognition. The paper presented a scheme to select key features using description based on mutual information method like mRMR as well as CMIM. Accuracy obtained from left iris code was $85.33 \%$ and right iris code was $84.33 \%$. The method is unachievable in case of person wearing glasses.

\section{HUMAN GENDER CLASSIFICATION}

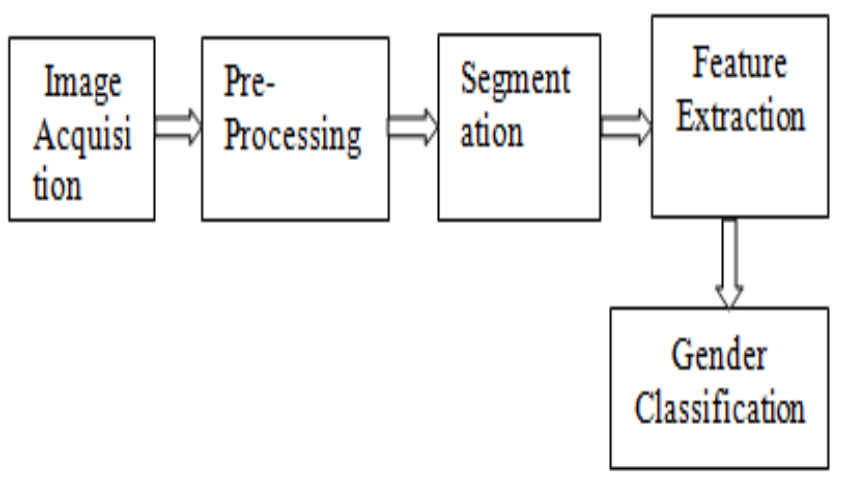

Fig.1 Block Diagram of human gender classification

The Fig.1 shows block diagram of human gender classification which includes Acquisition, pre-processing, feature extraction, classification.

Image Acquisition: It is the basic step of gender classification and aim is to obtain effective raw data.It is the process of creation of digitally encoded representation of visible properties of an object such as a physical scene or interior structure of object. Images are obtained by illuminating a scene and absorbing the energy reflected by the objects present in the scene. Different approaches are used to perform classification of gender, depends on acquired features.
Pre-Processing: Pre-processing is the essential procedure to upgrade the quality of raw data where not only training but also test images will be normalized to reduce noise from images. It includes various steps like grey scale conversion, histogram equalization and image resizing also. The Gaussian filter, median filter are used to remove noise present in the image. With proper signal processing steps, the irrelevant information is eliminated from raw data, improves the accuracy rate.

Segmentation: Segmentation is the process of dividing a digital image into multiple set of pixels also called as super pixels. It is used to simplify representation of image into significant manner. Segmentation is used to extract interest of area. The various algorithms are dependent on properties of intensity values like discontinuity as well as similarity algorithms.

Feature Extraction: The feature extraction method which is used to reduce the number of resources by extracting the features of the preprocessed information for classification. It is the process of dimensionality reduction which converts the raw information into manageable groups. Face feature is a technology used in a variety of applications that classifies human faces using digital images.

Machine learning is an application of artificial intelligence which provides capability to learn automatically and boost from experience without human assistance and adjust correspondingly. Learning includes observation or patterns present in the data and make well decision in future. Machine learning performance in a known task improves with the experience.

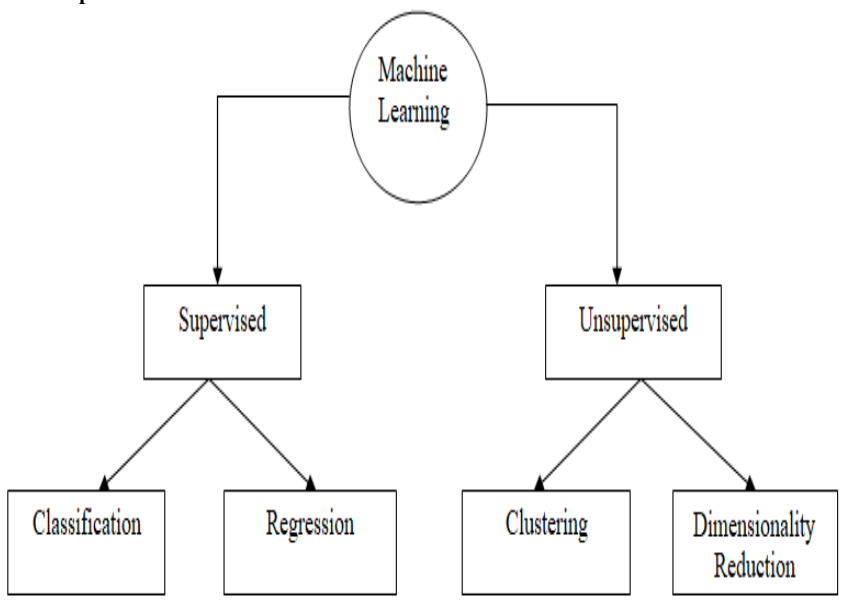

Fig.2 Machine Learning Methods

Some machine learning methods are -

Supervised learning, can apply on what has been learnt in past to predict new information using labeled data. Starting from known training dataset, algorithm creates a function to make prediction about output. Model is getting trained on the labeled dataset which consist of input and output parameters. Supervised learning is classified into two sub categories such as classification and regression. Classification is a supervised learning algorithm where output includes discrete values. It can be binary or multiclass classification in which binary classification predicts either yes or no but in multiclass, model 
predicts multiple classes. Regression is learning task where output consists of continuous values.

Unsupervised learning method is considerable when information is neither classified nor labeled (unknown, noisy data). Unsupervised learning studies, how system can conclude a function to represent a hidden structure from unknown data. Unsupervised learning is divided into two i.e. clustering and dimensionality reduction. Clustering is organization of unknown data into similar groups. Some clustering algorithms are k-means clustering, agglomerative clustering etc. For high dimensional data, dimensionality reduction is useful and purpose of this process is to reduce number of features under analysis. Dimensionality algorithms include Principle Component Analysis (PCA) and Linear Discriminant Analysis (LDA) and Non-negative matrix factorization (NMF) etc.

Gender classification: Gender classification is binary problem in which data is assigned as either male or female. Human able to classify gender mostly faces and information such as hairstyle, clothing, eyebrows, body shape and voice also. Now a day, clinical signal techniques have been proposed to help in the field of gender classification using EEG and electrocardiograph (ECG) signals.

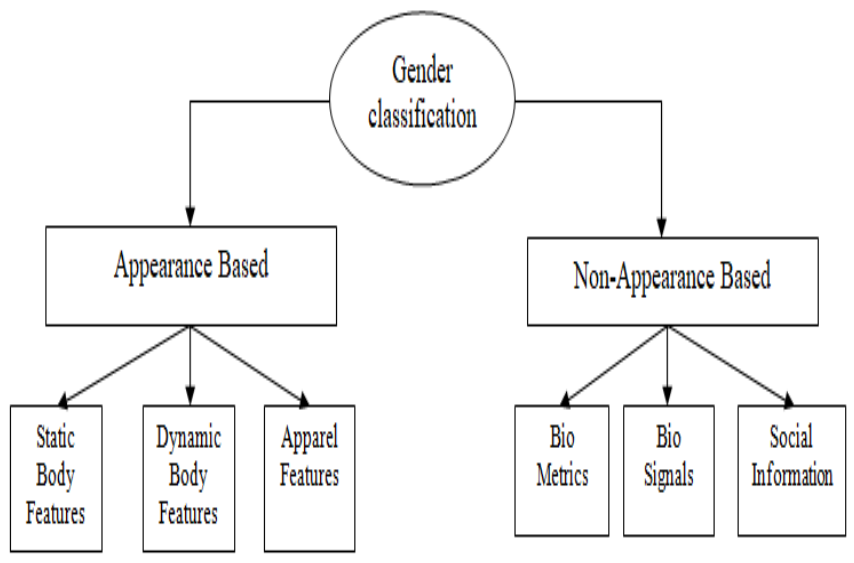

Fig.4 Gender Classification Approaches

Gender classification is divided into two categories such as appearance and non-appearance based approaches. One way to improve classification accuracy is to combine various features together to perform multi feature based gender classification. The appearance-based approach classifies gender based on the features obtained from the external information of human. It consists; static body feature like face, eyebrow, hand, body-shape, fingernail, etc., dynamic body features such as gesture gait, motion, etc. and apparel information feature include clothing, footwear, etc. The biological feature and daily social network data is used for non-appearance-based approaches. It includes biological features like fingerprint, voice, iris etc., bio signal features such as ECG, DNA etc. Social network information means information based on daily activities of individual includes email, blog and handwriting etc.

\section{CONCLUSION}

In a real-world, human gender classification using facial images is broadly accepted. This paper provides detailed review of a system for human gender classification using machine learning. This system tested with not only different standard databases but also videos. The system has successfully recognized classification with good accuracy and found less accurate in some algorithms. The misclassification of the similar features can be improved using system parameters with high recognition rate.

\section{REFERENCES}

11] Sandeep Kumar, Sukhwinder Singh and JagdishKumar, "Gender Classification Using Machine Learning with Multi-Feature Method",978-1-7281-0554-3/19/ @ 2019 IEEE

[2] Jinhyeok Jang, Hyunjoong Cho, Jaehong Kim, Jaeyeon Lee, andSeungjoon Yang, "Facial Attribute Recognition by RecurrentLearning With Visual Fixation",IEEE TRANSACTIONS ON CYBERNETICS, VOL. 49, NO. 2, FEBRUARY 2019.

[3] Juan E. Tapia And Claudio A. Perez, "Clusters of Features Using Complementary Information Applied to Gender Classification From Face Images",IEEEAccess, VOLUME 7, 2019.

4] George Azzopardi, Antonio Greco, Alessia Saggese And Mario Vento, "Fusion of Domain Specific and Trainable Features for Gender Recognition from Face Images",IEEE Access, VOLUME 6 2018

[5] Lin Yuan, Fanglin Chen, Ling-Li Zeng, Lubin Wang, and Dewen $\mathrm{Hu}$,"Gender Identification of Human Brain Image with A Novel 3D Descriptor",IEEE/ACM Transactions On Computational Biology And Bioinformatics, VOL. 15, NO. 2, MARCH/APRIL 2018.

[6] Ahmad Jamal KHDR and Cihan VAROL,"Age and Gender Identification by SMS Text Messages",978-1-5386-68788/18/@2018 IEEE.

[7] Juan E. Tapia, Claudio A. Perez and Kevin W. Bowyer, "Gender Classification from the Same Iris Code Used for Recognition", IEEE Transactions on Information Forensics and Security, Vol. 11, No. 8 , August 2016

[8] Jiale Chen, Sen Liu, Zhibo Chen, "Gender Classification In Live Videos",978-1-5090-2175-8/17/@2017 IEEE.

[9] Shannon Silessi, Cihan Varol, Murat Karabatak, "Identifying Gender from SMS Text Messages", 15th IEEE International Conference on Machine Learning and Applications, 2016.

[10] LahoucineBallihi, Boulbaba Ben Amor, Mohamed Daoudi,"Boosting 3-D-Geometric Features for Efficient Face Recognition and Gender Classification", IEEE Transactions On Information Forensics And Security, VOL. 7, NO. 6, DECEMBER 2012

[11] Huaining Cheng, and Kathleen M. Robinette, "Gender Recognition Using 3-D Human Body Shapes", IEEE Transactions on Systems, Man, and Cybernetics-Part C: Applications and Reviews, VOL. 41, NO. 6, NOVEMBER 2011.

[12] ErnoMakinen and RoopeRaisamo,"Evaluation of Gender Classification Methods with Automatically Detected and Aligned Faces", IEEE Transactions on Pattern Analysis and Machine Intelligence, VOL. 30, NO. 3, MARCH 200

\section{AUTHORS PROFILE}

Miss. Vaishnavi Yashwant Mali: She is M-Tech student of

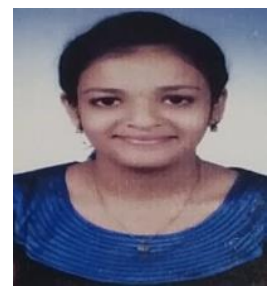
Walchand College of Engineering, Sangli, Maharashtra, India in Electronics. She completed her graduation in Electronics and Telecommunications from PVPIT, Budhgoan. She has keen interest in the field of Image Processing. 


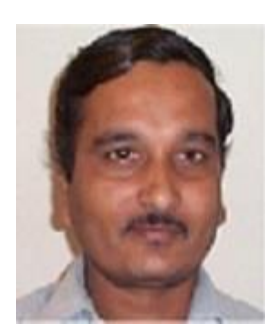

Dr. Babasaheb G. Patil: He received his M.E. Electronics degree in 1990 and B.E. Electronics in 1988 and Ph.D from Shivaji University, Kolhapur in 2015.He is currently working as Associate Professor indepartmentof Electronics, in Walchand College of Engineering, Sangli, Maharashtra, India.He has guided numerous students for graduation and Post-graduation. He is having keen interest in image processing and Communication. 